\title{
Serrated adenoma of the duodenum
}

\section{A Rubio}

The duodenum is the most common site for extracolonic adenomas in patients with familial adenomatous polyposis (FAP). These adenomas are usually tubular, villous, or tubulovillous. This report describes a patient with FAP who had a duodenal adenoma with a different histology-it had the microscopic attributes of a serrated adenoma-tumours that are relatively common in the colorectal mucosa and are occasionally found in the stomach. A 78 year old man with FAP and multiple adenomas was colectomised and the rectum amputated. Several years later he developed silent jaundice. The surgical specimen showed an adenomatous growth juxtaposing the papilla of Vater. The adenoma had epithelial fronds with crenated, sawtooth-like configurations caused by scalloped epithelial infolding. The nuclei covering the notched fronds were pleomorphic, stratified, either cigar shaped with irregular chromatin deposits or vesicular shaped with a large nucleolus. One area showed involvement of a duct by neoplastic epithelium with extension into periluminal glands. No invasive carcinoma was present. Serrated adenomas differ from tubular and villous adenomas in their histological organisation and their initial pattern of cell proliferation and genotypic aberration. Increased awareness of the existence of serrated neoplasms in the duodenum may result in similar cases being reported in the future.

$\mathrm{T}$ he duodenum is the most common site for extracolonic adenomas in patients with familial adenomatous polyposis (FAP)..$^{1-6}$ Duodenal adenomas occur much less frequently in patients with no history of FAP. ${ }^{7}$

Sporadic duodenal adenomas are histologically classified according to their configuration into tubular, villous, or tubulovillous. ${ }^{7}$ FAP duodenal adenomas are also villous or tubulovillous; they usually gather around the periampullar region and frequently contain Paneth and endocrine cells. ${ }^{1} 24$ This last characteristic suggests a multipotential stem cell origin in the duodenal crypts.

Recently, while reviewing partial duodenectomy specimens we found one patient with FAP who had a duodenal adenoma with a histological configuration that differed from those previously described in the literature. It had the microscopic attributes of a sporadic serrated adenoma, a tumour that is relatively common in the colorectal mucosa, ${ }^{8}{ }^{9}$ and is occasionally reported in the stomach. ${ }^{10}$

\section{"Recently, while reviewing partial duodenectomy speci- mens we found one patient with FAP who had a duodenal adenoma with a histological configuration that differed from those previously described in the literature"}

Recently, Matsumoto and colleagues ${ }^{11}$ reported serrated adenomas of the colon in three patients with FAP, and Gallagher and Phillips ${ }^{12}$ reported colonic serrated adenomas in eight patients with FAP.
Because no case of serrated adenoma in the duodenum in patients with FAP (or in sporadic cases) is on record in the literature, and taking into account the current interest in that adenoma phenotype in other mucosa of the gastrointestinal tract, we thought that it would be of interest to describe and to illustrate that case.

\section{CASE REPORT}

\section{The patient}

The patient was a 78 year old man with a diagnosis of FAP. Because of multiple colonic adenomas, a colectomy with ileorectal anastomosis was performed in 1967. The surgical specimen showed multiple colonic adenomas but no invasive carcinoma. In 1981, the rectum was amputated because of multiple adenomas with high grade dysplasia. Following annual examinations, he developed a silent jaundice in 1989. A percutanecus transhepatic cholaniography showed a $2 \mathrm{~cm}$ long stricture in the distal choledocus. At endoscopy, a $1.5 \mathrm{~cm}$ polyp juxtaposing the papilla of Vater was found. Endoscopic biopsies showed a tubulovillous adenoma with high grade dysplasia. Biopsies taken from other lesions in the duodenum were diagnosed as adenomas with high grade dysplasia. Endoscopic retrograde cholangio-pancreatography demonstrated a narrow choledocus near the papilla, and a dilated proximal choledocus. A Whipple operation was performed.

\section{Pathology}

The surgical specimen consisted of a partial gastrectomy ( $8 \mathrm{~cm}$ long along the greater curvature) with duodenectomy $(23 \mathrm{~cm})$, and a partial pancreatectomy $(7 \times 5 \times 3 \mathrm{~cm})$. A $10 \mathrm{~cm}$ long gallbladder with a $7 \mathrm{~cm}$ long common bile duct was included in the specimen.

At gross examination, a $1.5 \times 1.5 \mathrm{~cm}$ papillary tumour juxtaposing the papilla duodeni major was found. No gross tumour could be found in the ampulla of Vater or in the distal common bile duct. The bile duct was narrowed, and its proximal aspect was dilated. Twelve small polyps $(2-7 \mathrm{~mm})$ were found in the duodenal mucosa, proximal and distal to the tumour.

At histology, the tumour juxtaposing the papilla of Vater (papilla duodeni major) showed an adenomatous growth with a predominantly $(>80 \%)$ crenated, sawtooth-like configuration as a result of scalloped epithelial infolding (figs 1-2). The nuclei covering those serrated notched fronds were pleomorphic, stratified, either cigar shaped with irregular chromatin deposits or vesicular shaped with a large nucleolus. In other cells, the nucleoli were dark and irregular as a result of nucleolus associated chromatin. Some glands had a back to back arrangement, others a gland within glands arrangement. Mitotic figures were increased. One area showed involvement of a bile duct by neoplastic epithelium, with extension into the periluminal glands (fig 3). No invasive carcinoma was seen. The mucosa surrounding the tumour juxtaposing the papilla of Vater was of duodenal type

Abbreviations: FAP, familial adenomatous polyposis 


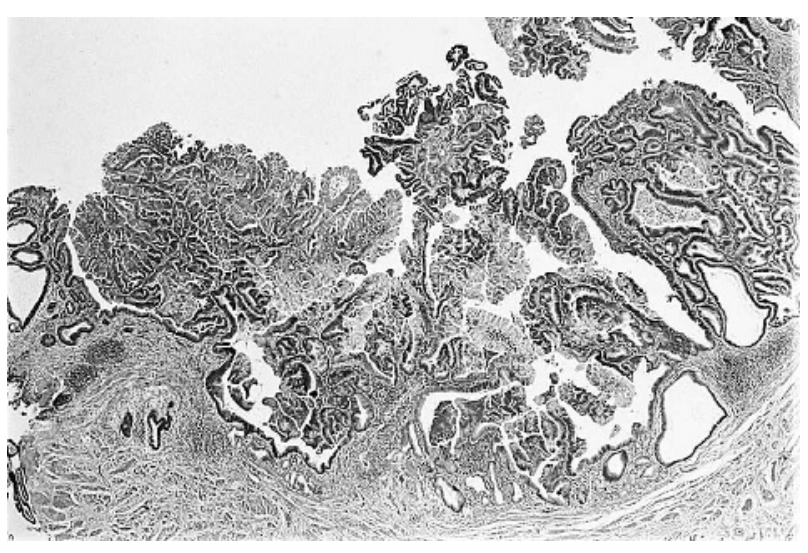

Figure 1 Low power view of a serrated adenoma in the duodenum in a patient with familial adenomatous polyposis (haematoxylin and eosin stain; original magnification, $\times 2$ ).

with a few Brunner glands. The remaining part of the adenoma showed tubular structures.

Although no tumour could be discerned in the ampulla of Vater or in the distal common bile duct at gross inspection, histological examination of the mucosa of the ampulla of Vater near the papilla showed a slightly serrated configuration with low grade dysplasia. This was interpreted as an extension of the adenomatous growth into the papilla of Vater. The distal common bile duct showed chronic inflammation, had cystically dilated glands, and showed pronounced fibrosis. No tumour was found.

Immunohistochemical stain of the duodenal tumour showed pronounced cellular proliferation (Mibl antibody; Dako, Glostrup, Denmark) in the serrated structures (fig 4).

Sections from the 12 duodenal polyps revealed five tubular adenomas, two villous adenomas, and five tubulovillous (mixed) adenomas. Two of the 12 adenomas had high grade dysplasia. Seven regional lymph nodes showed no metastasis at histology.

Haematoxylin and eosin stained sections from the serrated adenoma were observed in a fluorescent microscope, but no Paneth cells were found using that simple method of examination. ${ }^{13}$ The absence of Paneth cells was confirmed by the aid of lysozyme immunostaining. ${ }^{13}$ Chromogranin A and synaptophysin immunostaining showed no endocrine cells. The 12 adenomas present in the duodenectomy showed Paneth cells and endocrine cells.

In November 1995 the patient noticed a polyp at the ileostomy. That polyp was resected, and the histological examination revealed a radically excised mixed serrated $(60 \%) /$ villous $(40 \%)$ adenoma with high grade dysplasia and suspected invasion.

New polyps in the ileostomy were detected in December 1998 and in January 1999. Both polyps were removed, and the histology showed tubular adenomas with low grade dysplasia. Since then, minor polyps have evolved in the same area but they have not been removed.

The patient is well to this date (January 2004).

\section{DISCUSSION}

In FAP, adenomas are often found in the upper gastrointestinal tract. ${ }^{1-6}$ Duodenal adenomas have been reported in $24-93 \%$ of patients with FAP, usually later in the course of the disease. ${ }^{56}$ Most of these lesions have a predilection for clustering around the papilla of Vater. The lifetime risk of developing duodenal or periampullary cancer is $3-4 \%{ }^{6}$ The risk is up to 300 times greater than that of the general population, in which duodenal carcinoma is rare. ${ }^{37}$

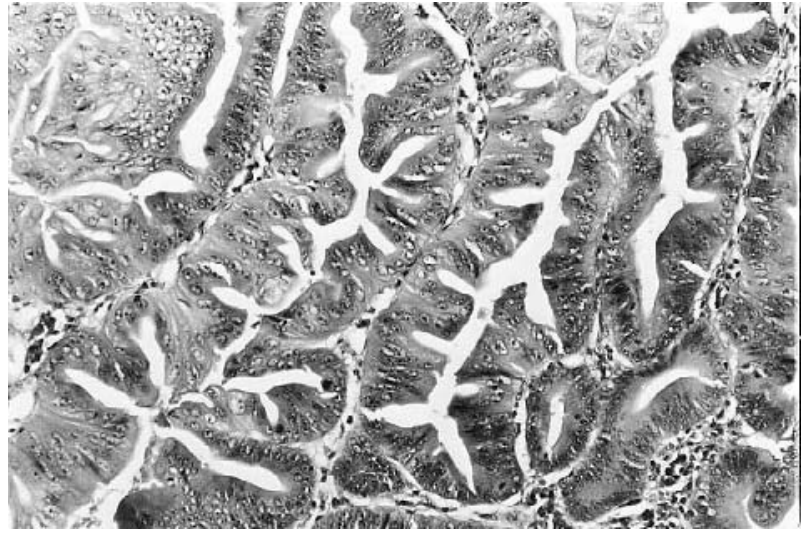

Figure 2 Detail from the duodenal serrated adenoma in fig 1 showing elongated fronds with lateral crenate, sawtooth-like notches as a result of scalloped epithelial indentations. Note carcinoma in situ (intraepithelial carcinoma) in the serrated fronds (haematoxylin and eosin stain; original magnification, $\times 25$ ).

All FAP and sporadic duodenal adenomas have been found at histology to be tubular, villous, or tubulovillous (mixed). ${ }^{1-6}$ Previous to this report, no cases of serrated adenoma of the duodenum have been recorded in patients with FAP, and in our department no serrated adenomas have been found during the 11 year period (from 1993 to 2003) that we have investigated duodenal biopsies from 240 patients with FAP who have multiple duodenal adenomas. Paradoxically, serrated adenomas in the duodenum should be common, because normal villi in the duodenal mucosa (even in patients with FAP) have serrated fronds. ${ }^{14}$

In our case, and according to the Vienna classification, ${ }^{8}$ areas with dark cigar shaped "picket fence" dysplastic nuclei reaching the superficial aspect of the epithelium were regarded as high grade dysplasia, and areas with vesicular shaped nuclei carrying a large nucleolus reaching the upper border of the epithelium were regarded as carcinoma in situ (intraepithelial carcinoma). ${ }^{15}$

Cellular proliferation (as assessed by Ki67) was intense in the dysplastic cells covering serrated indentations. The cellular proliferation in this serrated adenoma mimics that of serrated adenomas of the colon, which are much more common. ${ }^{9}$ In fact, in tubular, villous, and mixed (tubulovillous) adenomas cell proliferation occurs initially in the superficial (luminal) cells, whereas in serrated adenomas cell proliferation takes place in the cells at the bottom of the serrated structures. ${ }^{16}$

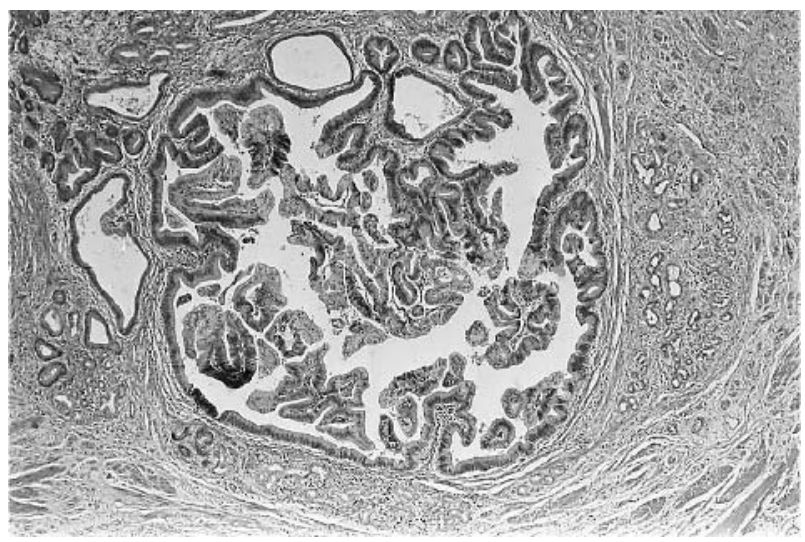

Figure 3 Involvement of a duct by neoplastic epithelium with extension into the periluminal glands. Note the presence of serrated adenomatous structures (haematoxylin and eosin stain; original magnification, $\times 10$ ). 


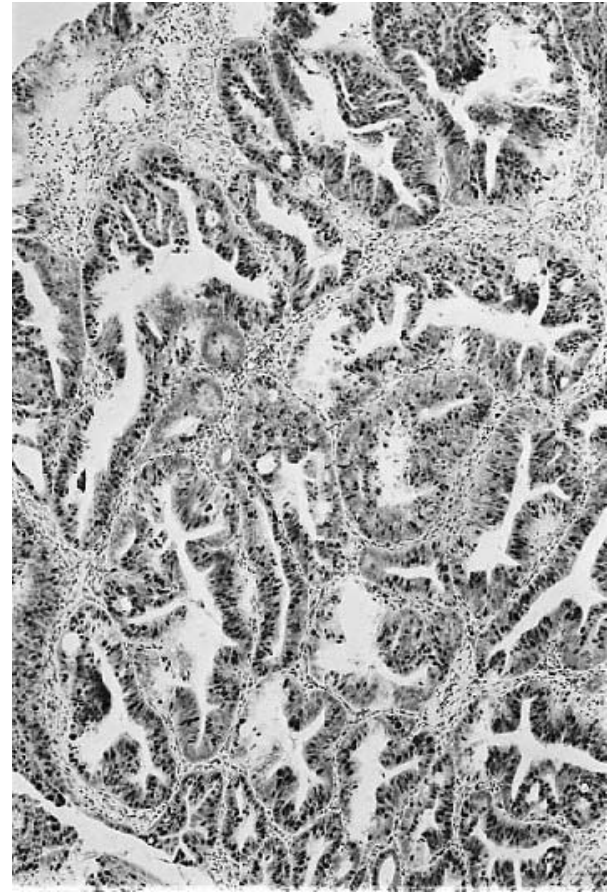

Figure 4 Immunohistochemical study showing pronounced cell proliferation in the serrated indented epithelium (Mibl antibody; original magnification, $\times 10$ ).

Expression of the p53 protein was not detected. However, it should be pointed out that the preparation had been fixed in formalin for two days. It is possible that the relatively long period of fixation led to spurious results when the sections were stained for $\mathrm{p} 53$.

"The structural and cell proliferative attributes of this neoplastic growth indicate that serrated adenoma is a special adenoma phenotype, at variance with tubular, villous, or tubulovillous adenomas"

Odze et al found Paneth cells in 68 of their 74 periampullar and duodenal adenomas from 30 patients with FAP. ${ }^{4}$ Their findings led to the suggestion that duodenal adenomas in FAP arise from the neoplastic transformation of an undifferentiated stem cell that retains its capacity for multidirectional differentiation. In our present case, neither Paneth cells nor endocrine cells could be demonstrated.

The structural and cell proliferative attributes of this neoplastic growth indicate that serrated adenoma is a special adenoma phenotype, at variance with tubular, villous, or tubulovillous adenomas. The finding that FAP adenomas may display different histological phenotypes both in the duodenum and in the colon ${ }^{11}{ }^{12}$ suggests that the genetic trait that triggers the development of thousands of adenomas may be unrelated to the molecular stimuli that generate the
Take home messages

- We report for the first time a case of serrated adenoma in the duodenum of a patient with familial adenomatous polyposis

- Increased awareness of the existence of serrated tumours in the duodenum may result in similar cases being reported in the future

etching of different architectural dissimilarities (tubular, tubulovillous, villous, or serrated) in those adenomas.

In conclusion, the first case of serrated adenoma in the duodenum in a patient with FAP is reported. Increased awareness of the existence of serrated tumours in the duodenum may result in similar cases being reported in the future.

Correspondence to: $\operatorname{Dr}$ C A Rubio, Gastrointestinal and Liver Pathology, Research Laboratory, Karolinska Institute, 17176 Stockholm, Sweden; Carlos.Rubio@onkpat.ki.se

Accepted for publication 2 February 2004

\section{REFERENCES}

1 Domizio P, Talbot I, Spigelman A, et al. Upper gastrointestinal pathology in familial adenomatosis polyposis: results from a prospective study of 102 patients. J Clin Pathol 1990:43:738-43.

2 Groves C, Saunders B, Spigelman A, et al. Duodenal cancer in patients with familial adenomatosis polyposis (FAP): results of a 10 year prospective study. Gut 2002;50:636-41.

3 Heiskanen I, Kellokumpu I, Järvinen H. Management of duodenal adenomas in 98 patients with familial adenomatosis polyposis. Endoscopy 1999:31:412-16.

4 Odze R, Gallinger S, So K, et al. Duodenal adenomas in familial adenomatosis polyposis: relation of cell differentiation and mucin histochemical features to growth pattern. Mod Pathol 1994:7:376-84.

5 Spigelman A, Williams C, Talbot I, et al. Upper gastrointestinal cancer in patients with familial adenomatosis polyposis. Lancet 1989; ii:783-5.

6 Vos tot Nederveen, Cappel W, Järvinen H, et al. Worldwide survey among polyposis registries of surgical management of severe duodenal adenomatosis in familial adenomatosis polyposis. Br J Surg 2003;90:705-10.

7 Seifert E, Schulte F, Stolte M. Adenoma and carcinoma of the duodenum and papilla of Vater: a clinicopathologic study. Am J Gastroenterol 1992;87:37-42.

8 Schlemper RJ, Riddell, Kato $Y$, et al. International consensus on the classification of gastrointestinal epithelial neoplasia. The Vienna classification. Gut 2000;47:251-5.

9 Rubio CA, Nesi G, Kato Y. Serrated and microtubular adenomas of the colon and rectum. An 8-year histological survey. Anticancer Res 2003;23:1693-6.

10 Rubio CA. Serrated neoplasias of the stomach: a new entity. J Clin Pathol 2001;54:849-53.

11 Matsumoto T, lida M, Kobori Y, et al. Serrated adenoma in familial adenomatous polyposis: relation to germline APC gene mutation. Gut 2002;50:402-4.

12 Gallagher M, Phillips R. Serrated adenomas in FAP [letter]. Gut 2002;51:895-6.

13 Rubio CA, Nesi G. A simple method to demonstrate normal and metaplastic Paneth cells in tissue sections. In Vivo 2003;17:67-72.

14 Sternberg S. Small intestine. In: Sternberg S, eds. Histology for pathologists. Philadelphia: Lippincott-Raven, 1997:498-500.

15 Rubio CA. Gastrointestinal epithelial neoplasia. Gut 2003;52:455-6.

16 Rubio CA, Rodensiö M. p53 overexpression in flat serrated adenomas and flat tubular adenomas of the colorectal mucosa. J Cancer Res Clin Oncol 1995; 121:571-6. 